\title{
Stimulus effects on cancellation task performance in children with and without dyslexia
}

\author{
Ho-Chuan Huang \\ National Kaohsiung University of Applied Sciences, Kaohsiung City, Taiwan \\ AND \\ Tsui-Ying WANG \\ National Cheng Kung University, Tainan City, Taiwan
}

\begin{abstract}
Both the sensitivity and administration time of a test are important in evaluating visuospatial attention in clinical settings, especially with respect to external validity. The purpose of the present study was to propose an adaptive model that provides a reference for test modification by manipulating target-to-distractor (T/D) ratios and the number of stimuli on the computerized cancellation test system. Tasks with different T/D ratios and numbers of stimuli were presented to two groups — children with and without dyslexia $(n=41$ and 65 , respectively) - to determine whether their visuospatial attention performance differed on different test forms. In general, there were significant differences between the two groups in hit rates, completion times, and performance quality (PQ) scores. The PQ score of visual attention was affected by the T/D ratios rather than by the number of stimuli. The findings suggested that the T/D ratio has a strong effect on PQ scores, and that it should be taken into consideration in test and task design.
\end{abstract}

Cancellation tasks are widely used in clinical and research settings to screen selective attention, visual search abilities, and visuospatial dysfunction (Dawson \& Tanner-Cohen, 1997; Lezak, 1995; Quintana, 1995). Although various formats have been developed for different research purposes, all cancellation testing involves the scanning, identifying, and marking out of specific target stimuli in a given visual display. Success is determined by the number of omissions and correct responses, and by the completion time for the task. Disproportionately scattered responses, or those on only one side of the paper, may indicate visual attention problems or hemineglect (Lezak, 1995). People with hemineglect often show reduced awareness of stimuli on their left side. Some studies have proposed that children with dyslexia often have insufficient processing of the stimulus on the left visual field on cued tasks (Heiervang \& Hugdahl, 2003) or on line-bisection tasks (Sireteanu, Goertz, Bachert, \& Wandert, 2005). It is widely accepted that children with dyslexia may have visual attention deficits (Stein \& Walsh, 1997; Vidyasagar \& Pammer, 1999). However, cancellation tasks have rarely been used in dyslexics to explore how they attend to stimuli in the visual field, or to understand their visual attention deficits as well as their hemispatial performance.

There are various cancellation-task designs for different research and clinical needs. Letter- and number-cancellation tasks with structured arrays or long string forms, such as the Cs and Es task (Diller, Ben-Yishay, Gerstman, Goodkin, \& Weinberg, 1974) and the 2s and 7s task (Ruff, Evans, \& Light, 1986) use the constraints of both time and scanning directions, and use accuracy as the measure of selective attention. Some symbol- and letter-cancellation tasks with complex or random arrays do not use time and scanningdirection constraints, such as the Bells test (Gauthier, Dehaut, \& Joanette, 1989), the Star test (Halligan, Wilson, \& Cockburn, 1990), the symbol cancellation test (Weintraub \& Mesulam, 1988), and the letter cancellation test (Geldmacher, 1996, 1998). These tasks simply provide results for outcome measures with little discussion about scanning paths for clinical judgment of visuospatial inattention. However, neither response accuracy nor processing speed exclusively in such tasks can be quantified as a single measure for task performance. Performance quality (PQ) scoring that accounts for both speed and accuracy to represent overall quality of visuospatial attention has been addressed (Geldmacher, 1996). PQ scoring is a supplemental method that provides an objective measure for comparing cancellation performances with different speeds.

Performance on cancellation tasks may be influenced by stimulus and task factors (Byrd, Touradji, Tang, \& Manly, 2004; Hogeboom \& van Leeuwen, 1997; Scharroo, Stalmeier, \& Boselie, 1994). Stimulus factors include the number of stimuli, the absence or presence of distractors, perceptual similarity, the target-to-distractor (T/D) ratio, stimulus encoding (e.g., verbal or nonverbal), and so on. Task factors include matrix size (the number of rows and columns), matrix configuration (random vs. structured arrays), font size, space between the stimuli, the cancellation method, and so on. Geldmacher (1996) studied the per- 
formance of young adults on cancellation tasks: Data on the same task factors were grouped to compare the effects of different $\mathrm{T} / \mathrm{D}$ ratios and different numbers of stimuli. They suggested that $T / D$ ratios had a strong effect on the overall performance score. Mesulam (2000) reported that when the task difficulty is increased, the task becomes more sensitive for screening visual inattention problems. For example, it is more challenging for a patient with the perceptual deficit of neglect to detect letters than lines, and complex shapes than letters, as well as to search targets embedded in random arrays rather than in structured arrays. The challenge of perceptual discrimination is a major determinant of the level of task difficulty, one that also increases the need for attentional control (Geldmacher, 1996; Iles, Walsh, \& Richardson, 2000; Mesulam, 2000).

Feature integration theory explains the stimulus effect in visual search behavior (Treisman \& Gelade, 1980). If the feature of a target is distinct from the feature of the distractors (pop-out features), the target can be detected in the preattentive process, which requires minimal attentional control in identifying each target. The reaction times and accuracy of target identification remain constant despite the increase in the number of stimuli in the feature search task. On the contrary, if the target is defined by a conjunction of features, and if it shares one or more features with the distractors, then the target identification requires a serial search with more attentional control. However, conjunction features may "pop out," depending on the similarity of distractors and the difference between targets and distractors in the display (Duncan \& Humphreys, 1989).

Chatterjee, Thompson, and Ricci (1999) investigated the influences of spatial location, the number of stimuli, and target discrimination ability (i.e., $\mathrm{T}$ vs. $\mathrm{X}$, and $\mathrm{T}$ vs. $\mathrm{L}$ ) on the probability of canceling a target in neglect patients. Their study showed that patients with relatively mild neglect canceled more targets when the targets were more easily discriminated from distractors. In a study of adults without visuospatial deficits, Geldmacher (1996) concluded that the performance quality of the cancellation task is adversely affected by a higher proportion of distractors rather than by an increase in the number of stimuli. From the task aspect, more distractors may increase the potency of cancellation tasks to detect visuospatial deficits.

Because of the minimal testing time, training, and specialized equipment required by the cancellation paradigm, it has been widely used to study visuospatial attention in adults with or without pathological conditions. Except for pathological conditions, both age and maturity affect an individual's performance on cancellation tasks. Search time is related to the age-related processing speed that increases as a child matures, reaching its apex in early adulthood, and gradually declining with age (Trick \& Enns, 1998). Other mechanisms involved in visual search tasks - such as visual acuity, eye movement, shifting from one attention focus to another, encoding of visual stimuli, and cognitive control-also contribute to the progress of children's visual search ability. Studies about the progress of cancellation test performance in children reported that near-perfect accuracy was achieved by age 7 , whereas the speed and efficiency of performance continued to improve through early adolescence (Dumont, Stevens, Dawson, Guare, \& Weiler, 2001; Wasserman, Mitchell, \& Melrose, 1995). However, the effect of the number of stimuli on the cancellation-task performance in children with or without dyslexia remains unclear. Friedmann and Nachman-Katz (2004) reported a case of neglect dyslexia - someone who performed well in visuospatial tests (including cancellation tasks) but who showed deficits in word encoding and sentence reading. They also mentioned the lack of population studies for dyslexics. If we knew more about the effect of the number of stimuli on the visual search behavior of children with visuospatial dysfunctions, we might know whether manipulating that number increased or decreased the task sensitivity to cancellation tests, which would allow testers to screen out children with mild visuospatial dysfunctions. There is no published literature on this topic.

As children develop from early to late childhood, they spend less time and perform more accurately on cancellation tasks (Di Filippo et al., 2005; Dumont et al., 2001). Most school-age children without visuospatial dysfunctions use systematic search and have near-perfect accuracy on cancellation tasks (Wasserman et al., 1995). In comparison with children without dyslexia, children with developmental dyslexia may have prolonged attention-dwell time, difficulty shifting attention during visual or auditory processing, and less focused attention on visual tasks. Attention deficits as such are related to poor visual search performance on visual tasks (Casco \& Prunetti, 1996; Facoetti, Lorusso, Paganoni, Umiltà, \& Mascetti, 2003; Iles et al., 2000; Sireteanu, Goebel, Goertz, \& Wandert, 2006). Monitoring children's directed attention would help to identify the visual processing strategies that they use when comprehending their surroundings and reading materials. Various studies on visual attention have compared children with and without dyslexia by using different methods, such as visual scanning, cancellation tasks, and continuous performance tests (Hawelka \& Wimmer, 2005; Iles et al., 2000; Taroyan, Nicolson, \& Fawcett, 2007). Results have suggested that children with developmental dyslexia or attention deficits or both showed impaired performance in both their speed of and capacity for information processing. Slower reaction times and the larger number of response errors in visual search have been associated with the difficulties of dyslexic children in academic and life tasks (Casco \& Prunetti, 1996; Casco, Tressoldi, \& Dellantonio, 1998; Vidyasagar \& Pammer, 1999; Williams, Brannan, \& Lartigue, 1987).

Dyslexic children usually perform less accurately on visual attention tasks, and their reaction times are affected by size effects in conjunction search with serial processing (Casco \& Prunetti, 1996; Casco et al., 1998; Iles et al., 2000; Shalev \& Tsal, 2003). Iles et al. (2000) used tasks with different levels of similarity between targets and distractors, and different numbers of distractors in their experiments. They concluded that dyslexic children with early visual processing deficits also have visual attention problems related to parietal cortex dysfunction.

In contrast with adults, who tend to make more errors on the right side during cancellation tasks, children under school age make more errors and spend more time on the left side during visual search (Sireteanu et al., 2006; 
Sireteanu et al., 2005). Adults without visuospatial deficits tend to overestimate the left visual field because of the advantage of right hemispheric processing; however, individuals with hemineglect usually ignore their left extrapersonal space (Halligan \& Marshall, 1992), and those with dyslexia process stimuli in the left visual field more slowly (Hari, Renvall, \& Tanskanen, 2001). In previous studies (Hari et al., 2001; Roeltgen \& Roeltgen, 1990; Sireteanu et al., 2005), it has been suggested that people with developmental dyslexia showed a left "minineglect"-an immature attention pattern due to the right parietal lobe hypofunction caused by a magnocellular deficit. However, this notion needs to be further clarified by examining spatial organization in visual attention tasks.

We designed four cancellation forms with different numbers of stimuli and T/D ratios in a computerized cancellation test system to investigate whether the target detection performance would be different on different forms. We hypothesized that (1) there would be target-detection score differences (i.e., the hit rates, task completion times, and PQ scores) between the dyslexic and control groups; (2) there would be the target-detection score differences among the four forms; and (3) the control group would show a left hemispatial advantage, but the dyslexic group would show no left-right difference. Therefore, we compared target-detection performance on different forms and examined the lateral spatial performance in both groups.

\section{METHOD}

\section{Participants}

We recruited 106 children between the ages of 10 and 12 years. The dyslexic group consisted of 41 children ( 34 boys [ $82.9 \%$ ] and 7 girls [17.1\%]), and the control group consisted of 65 children (50 boys [76.9\%] and 15 girls [23.1\%]). The children in the dyslexic group were recruited through local education authorities and had already undergone a well-documented screening and decision process for the diagnosis of dyslexia that had been conducted by a special education task force. The dyslexic children had average IQs but poor school achievement and reading difficulties. Children in the control group were not dyslexic, nor did they have any other learning difficulties. The participants in both groups were in elementary school grades 4,5 , or 6 . Children with any neurological disease, physical illness, visual or motor problems, emotional disturbance, or any conditions of mental retardation were excluded from the present study.

\section{Instruments}

We created four symbol cancellation forms based on a symbol cancellation test (Weintraub \& Mesulam, 1988); all of the forms were in random arrays, but they varied in the number of stimuli and T/D ratios. Forms 1 and 2 had 120 stimuli each, and Forms 3 and 4 had 240. Forms 1 and 3 had a 1:4 T/D ratio, whereas Forms 2 and 4
Table 1

Characteristics of the Four Test Forms

\begin{tabular}{cccc}
\hline Forms & $\begin{array}{c}\text { Number of } \\
\text { Stimuli }\end{array}$ & T/D Ratio & $\begin{array}{c}\text { Number } \\
\text { of T/D }\end{array}$ \\
\hline 1 & 120 & $1: 4$ & $24 / 96$ \\
2 & 120 & $1: 9$ & $12 / 108$ \\
3 & 240 & $1: 4$ & $48 / 192$ \\
4 & 240 & $1: 9$ & $24 / 216$ \\
\hline
\end{tabular}

Note $-\mathrm{T} / \mathrm{D}$, target/distractor.

had a 1:9 T/D ratio (Table 1). The tests were administered on a tablet computer (screen size: $9 \times 12$ in.) with a computer-assisted testing procedure. There were an equal number of stimuli in each Cartesian quadrant, with “涼” as the target.

\section{Procedures}

The participants took the four cancellation subtests in a counterbalanced order to eliminate the practice effect. They were given detailed instructions and practice trials until they felt ready for the task. A cordless responsive pen was used for data input (stimulus marking) on the tablet screen, which resembled the canceling process on paper. The computer-assisted testing procedure automatically crossed out each selected stimulus with a blue " $\mathrm{X}$ " and synchronously recorded outcome data (e.g., time-stamped $x$ - and $y$-coordinates) in the database. Completion time was not limited, but participants were instructed to complete the task as quickly as possible without sacrificing accuracy. When finished, participants could press any key on the keyboard to terminate the test. The tablet was placed in front of the participant at a $30^{\circ}$ angle and $20 \mathrm{~cm}$ from the edge of the table. Head and eye movements were not restricted during the test.

Raw outcome data, such as the number of correct responses, omissions and commissions, and task completion times were recorded by the computer. The accuracy was represented by "hit rate," which was defined as the percentage of correct hits, and commissions were represented by "error responses." Considering the data distribution pattern and overall quality of visuospatial attention, we used the PQ score to measure visual attention performance - a numerical measure of visuospatial attention that accounts for both speed and accuracy (Geldmacher, 1996). The formula for calculating the PQ score is

$$
\text { PQ score }=\frac{\text { correct responses }}{\text { total target }} \times \frac{\text { correct responses }}{\text { completion time }} \text {. }
$$

The hit rate, completion time, and PQ score were dependent variables (i.e., outcome measures). The error distributions (the number of error responses) on the left and right sides were represented by the counted frequencies. The errors on the left and the right for the four forms were compared separately for each group. The "initial location," classified as either "left" or "right," was the location of the first stimulus marked by the participant.

\section{RESULTS}

The means and standard deviations were computed for the three dependent variables (Table 2). The form effects

Table 2

Descriptive Data for Dependent Variables for Both Groups

\begin{tabular}{|c|c|c|c|c|c|c|c|c|c|c|c|c|}
\hline \multirow[b]{3}{*}{ Form } & \multicolumn{4}{|c|}{ Hit Rate $(\max .=1)$} & \multicolumn{4}{|c|}{ Time (sec) } & \multicolumn{4}{|c|}{ PQ Score } \\
\hline & \multicolumn{2}{|c|}{ Control } & \multicolumn{2}{|c|}{ Dyslexic } & \multicolumn{2}{|c|}{ Control } & \multicolumn{2}{|c|}{ Dyslexic } & \multicolumn{2}{|c|}{ Control } & \multicolumn{2}{|c|}{ Dyslexic } \\
\hline & $M$ & $S D$ & $M$ & $S D$ & $M$ & $S D$ & $M$ & $S D$ & $M$ & $S D$ & $M$ & $S D$ \\
\hline 1 & .981 & .039 & .948 & .095 & 38.01 & 11.10 & 48.59 & 15.63 & .633 & .201 & .517 & .155 \\
\hline 2 & .988 & .038 & .965 & .055 & 31.02 & 10.11 & 36.22 & 13.66 & .398 & .132 & .352 & .127 \\
\hline 3 & .965 & .046 & .935 & .094 & 73.01 & 25.06 & 98.89 & 36.92 & .639 & .197 & .495 & .163 \\
\hline 4 & .970 & .037 & .924 & .084 & 58.80 & 18.36 & 75.05 & 31.27 & .407 & .133 & .341 & .122 \\
\hline
\end{tabular}

Note $-n=65$ for the control group and $n=41$ for the dyslexic group; PQ Score, performance quality score. 
Table 3

Univariate Analysis of Between- and Within-Subjects Effects

\begin{tabular}{llrccc}
\hline & \multicolumn{1}{c}{ Source } & $d f$ & $F$ & $\eta$ & $p$ \\
\hline \multirow{4}{*}{ Hit rate } & Form & Between Subjects \\
& Form $\times$ group & 3 & 13.867 & .27 & $<.001$ \\
& Error & 312 & 0.996 & .09 & .39 \\
Time & Form & 3 & 311.119 & .95 & \\
& Form $\times$ group & 3 & 10.92 & .15 & $<.001$ \\
& Error & 312 & $(S S=56,139.7)$ & .48 & \\
PQ score & Form & 3 & 3.891 & .78 & .001 \\
& Form $\times$ group & 3 & 0.153 & .16 & $<.001$ \\
& Error & 312 & $(S S=2.201)$ & .59 & \\
& & Within Subjects & & \\
Hit rate & Group & 1 & 13.867 & .34 & $<.001$ \\
& Error & 104 & $(S S=.829)$ & .93 & \\
Time & Group & 1 & 16.73 & .37 & $<.001$ \\
& Error & 104 & $(S S=131,005.7)$ & .93 & \\
PQ score & Group & 1 & 10.672 & .31 & .001 \\
& Error & 104 & $(S S=8.438)$ & .95 & \\
\hline
\end{tabular}

Note-PQ score, performance quality score; $S S$, sum of squares.

on the dependent variables for the control and dyslexic groups were tested using a MANOVA with repeated measures. In this approach, the between-subjects factor was "group" (dyslexic and control groups), and the withinsubjects factor was "form" (four levels). Where a significant $F$ value was obtained, we conducted a post hoc analysis for multiple comparisons. There were significant main effects for group [Wilk's $\lambda=.765, F(3,102)=10.425$, $p<.001$ ] and for form [Wilk's $\lambda=.043, F(9,96)=$ $237.587, p<.001]$, and there was a group $\times$ form interaction [Wilk's $\lambda=.732, F(9,96)=3.898, p<.001$ ].

Table 3 shows the univariate results for the dependent variables within each significant main effect and interaction. The group effect was significant for the three dependent variables. The dyslexic group had significantly lower scores for hit rate, task completion time, and PQ score than did the control group. The form effects for the dependent variables were the term of interest. Information on how these dependent variables are affected by forms implies the ability to adjust test difficulty to improve test sensitivity. The form $\times$ group interaction was significant for completion time and PQ score, but there was no effect for hit rate. Therefore, we compared hit rates on each form. Because of the significant interaction, we made multiple comparisons between forms as well as compared group differences for completion times and PQ scores. The $\alpha$ was corrected to $.0125(0.5 / 4)$ for multiple comparisons with Bonferroni adjustments.

For the hit rate, the two groups had significant discrepancies on Form $4(p<.001)$, but other differences were not significant $(p>.0125)$. For hit rates on different forms, we found no significant differences in the hit rates between Forms 1 and 2 (with 120 stimuli) or between Forms 3 and 4 (with 240 stimuli) (see Figure 1). That is, when the number of stimuli was fixed, the hit rate was unaffected by different T/D ratios. The hit rate on Form 2 was lower than those on Form $3(t=-3.82, p<.001)$ and Form $4(t=-4.24, p<.001)$.
In terms of task completion time, except for no group difference on Form $2(p>.0125)$, the dyslexic group spent significantly longer than the control group on the rest of the forms $(p s \leq .001)$. For each group, the task completion times differed: They were ranked as Form $2>$ Form $1>$ Form $4>$ Form 3 (Figure 2). Both the T/D ratios and the number of stimuli affected task completion time $(p s<.001)$. We found that more stimuli required more time to process, and that when the number of stimuli was fixed, the tasks with a higher T/D ratio required more time to complete.

A post hoc analysis showed significant differences in PQ scores on three forms for the two groups (Form 1, $p=$ .002 ; Form $3, p=.000$; Form $4, p=.013)$ but no significant differences on Form $2(p=.080)$. For each group, the $\mathrm{T} / \mathrm{D}$ ratio had a significant effect on the PQ scores on all forms $(p s<.001)$ (Figure 3$)$. When the number of stimuli was fixed, a high PQ score was associated with a higher $\mathrm{T} / \mathrm{D}$ ratio. When the T/D ratio was fixed at $1: 4$ or $1: 9$, the PQ scores were not affected by different numbers of stimuli $(120,240)$.

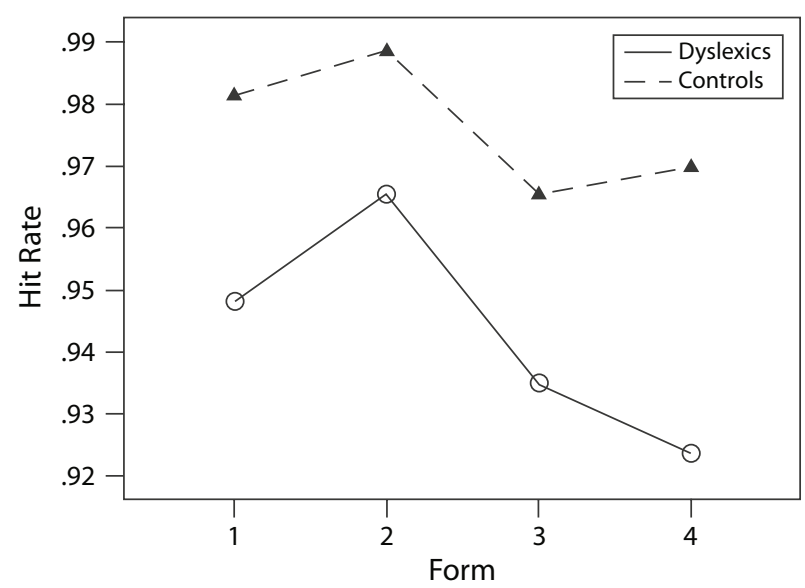

Figure 1. Hit rate for the four forms for the two groups.

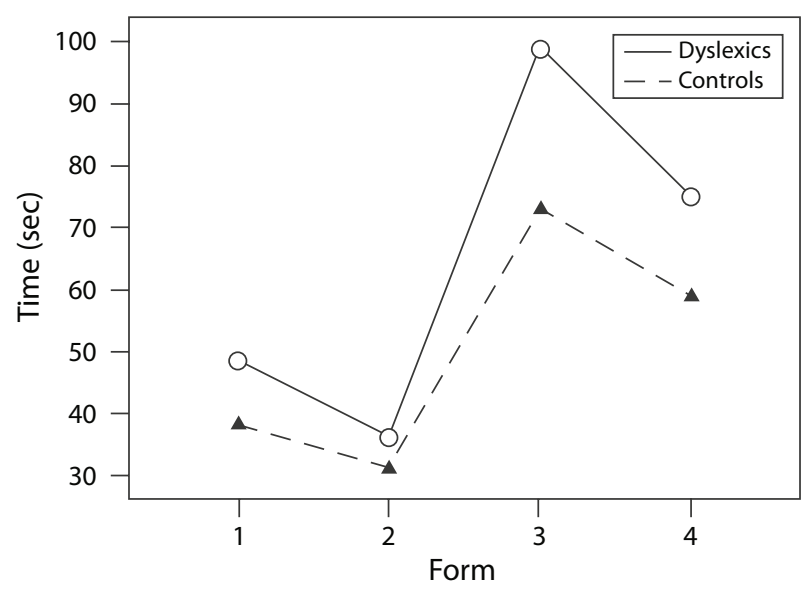

Figure 2. Task completion times for all four forms for the two groups. 


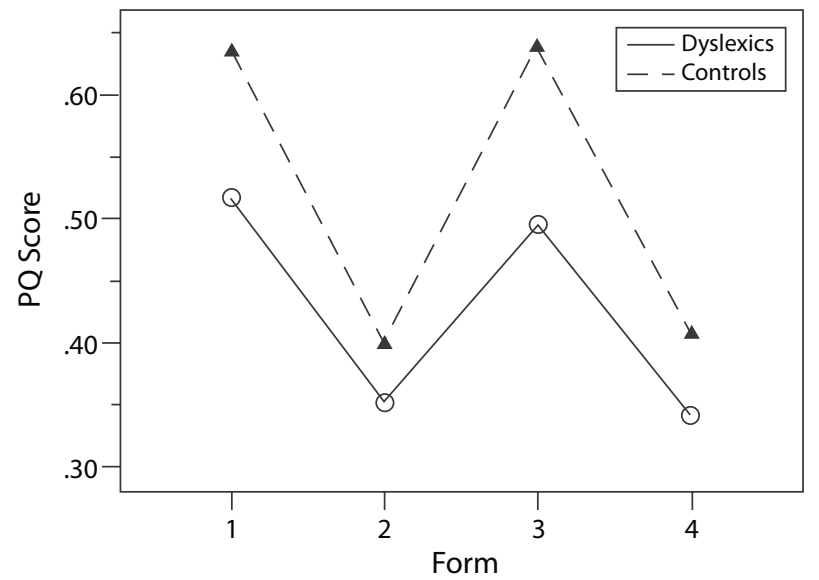

Figure 3. Performance quality (PQ) scores for all four forms for the two groups.

\section{Performance on the \\ Lateral Fields for Both Groups}

To determine whether the participants would perform more accurately on one side of the screen than on the other, we used paired $t$ tests to compare the left-right differences in the hit rates between the four forms for each group. Both groups had higher hit rates on the left side of Form 4 (dyslexic, $t=2.196, p<.05$; control, $t=2.243$, $p<.05$ ), but no significant left-right differences on the remaining three forms. Regardless of the grouping, more participants started their initial responses on the left side than on the right side on all cancellation forms $(p<.001$ for each). The frequencies of the initial responses from the left and right sides were the same between groups except for Form 2, on which initial responses were initiated on the left more frequently in the control group than in the dyslexic group $(\phi=.198, p<.05)$.

\section{DISCUSSION}

We manipulated the T/D ratio and the number of stimuli in the symbol cancellation forms in order to understand their impact on the outcome measures. We found that the higher hit rate was associated with fewer stimuli, and that the higher PQ score was associated with a higher T/D ratio. More stimuli in the visual display meant that more time was required to finish the cancellation tasks. Our findings are consistent with those of Geldmacher (1996). Although these two studies differ in many respects - particularly in the number and type of stimuli, population age range, and cancellation task method (computerized vs. paper-and-pencil testing) - Geldmacher (1996) reported a strong effect from the T/D ratio and no effect from the number of stimuli on the PQ score for random-letter cancellation tasks.

Our results suggest that when the number of stimuli was increased, response accuracy became more challenging and completion time increased. However, the ceiling effect of hit rate in both groups may restrict test sensitivity to minor dysfunction in visual attention. The control group had at least $96 \%$ hit rates on average, and the dyslexic group had at least $92 \%$ hit rates on average. If we look at the results of multiple $t$ tests for the two groups, only Form 4 shows a significant difference $(p<.001)$. The results suggest that the dyslexic group may have had a minor dysfunction in visual attention and that the problem might have become marked if the T/D ratio and the number of stimuli in a test form were increased.

The completion time results in both groups support the notion that cancellation tasks with targets amid distractors require serial search strategies for visual processing. The average completion time almost doubled when the number of stimuli doubled from 120 to 240 with the same T/D ratio (see Table 2). For Forms 1 and 2 (120 stimuli), the dyslexic group spent significantly more time than the control group to complete the tests, but the children in both groups showed a similar variation within group according to the standard deviations ( $S D \mathrm{~s})$. All $S D$ s of the completion times were large, and on Forms 3 and 4 (240 stimuli), the $S D$ s were even larger within each group. The children in the dyslexic group exhibited more individual differences than did those in the control group on the tasks with 240 stimuli, especially for Form 3 , which had more targets. Both groups spent the most time and had the lowest hit rates on Form 3. These findings may indicate that Form 3 was challenging for some children in this study and that it caused huge variations among them. Individual differences when challenged by a difficult task, such as Forms 3 and 4, may be the result of maturation differences. In one study (Kiliç, Sener, Koçkar, \& Karakas, 2007) of 6- to 11-year-olds who showed a huge time difference when completing paper cancellation tests, the $S D$ values accounted for more than one third of the mean time (e.g., symbol cancellation: $155.53 \pm 55.09 \mathrm{sec}$; letter cancellation: $201.63 \pm 91.46 \mathrm{sec}$ ). Similar to those in our study, these findings showed that when the tasks became more difficult (symbol to letter cancellations), the participants took more time, and that completion time differences also increased.

Since hit rate is not a sensitive index because of the ceiling effect, and since completion time may be wideranging because of individual variations, the PQ score is a good indicator of group difference. The PQ score considers both accuracy and time, and it indicates the speedaccuracy trade-off strategy used by the individual. We also observed that some children might quickly finish the test tasks and sacrifice accuracy, whereas some might spend a lot more time reviewing their answers. These impulsive and compulsive completion patterns may result from immature cognitive control of attention allocation and lead to poor performances on visual search tasks (Geldmacher, 1996). Summarizing from the aforementioned results, we suggest that a measure of impulsivity or compulsivity as a possible mediating variable would be helpful to justify its impact on outcome measures.

The dyslexic group had lower scores than the control group for hit rate, task-completion time, and PQ score on all cancellation forms. Casco et al. (1998) reported that selective attention is required to detect targets in a complex background and that attentional capacities must be deployed serially across the display. They suggested that reduced speed and a large number of visual errors in reading may be related to the selective visual attention deficits 
in children with poor visual search. Ben-Yehudah, Sackett, Malchi-Ginzberg, \& Ahissar (2001) addressed the impairment of the "retain-and-compare" mechanism in association with the selective attention deficits in dyslexic adults; they suggested the involvement of parietal deficits in dyslexia. In tasks that search for targets, either present or absent, among different set sizes of stimuli, previous studies (Iles et al., 2000; Sireteanu et al., 2006; Sireteanu et al., 2005; Vidyasagar \& Pammer, 1999) have shown that the dyslexic groups were less accurate and had longer reaction times, and that their performance was adversely affected by the number of distractors. These findings explain our results, which show that dyslexic children had deficits in managing tasks that require visual search ability, and that their performance was hampered by increased distraction and complexity in the task.

The dyslexic and control groups showed similar patterns for within-subjects performance for hit rate, taskcompletion time, and PQ score on the four cancellation tasks (Figures 1, 2, and 3). In general, the hit rate was not a sensitive indicator of different performances between the dyslexic and control groups in the present study. The dyslexic group took significantly longer than the control group to finish all tasks, except for Form 2. The PQ scores on Form 2 were not different between groups either. It seems that no matter which outcome measure was compared, Form 2 was not able to distinguish both groups well. On the other hand, even though the overall search of the dyslexic group was less efficient, their performance was task dependent. A cancellation task with fewer stimuli and a larger T/D ratio (e.g., 1:4) may allow children with dyslexia to more easily identify the correct targets. The implication of such findings is that a modified task environment may allow dyslexic children to process reading tasks better by providing less visual complexity, fewer irrelevant distractions for attention, and a reasonable time frame for learning. This compensatory strategy may improve chances for success on visual tasks. In addition, tests such as Form 3-with a higher T/D ratio and more stimuli- took longer to complete (Figure 2). Such cancellation tasks may correlate with the ability of sustained attention, but easy and short cancellation tasks may not. Further correlation studies with other attention tests are needed to substantiate this observation.

We found that hit rates on the left and right for different tasks were inconsistent. This finding is similar to those in previous studies (Gauthier et al., 1989; Geldmacher \& Alhaj, 1999; Geldmacher, Doty, \& Heilman, 1994; Weintraub \& Mesulam, 1988) that reported inconclusive findings for the error distributions on the left versus the right side. Some arguments to explain these inconsistent findings have been proposed: One is different reading habits within different populations; another is verbal- versus nonverbal-hemisphere processing in different populations. We report inconsistent hit-rate differences between the left and right sides among the four tasks, which varied in task complexity (both the number of stimuli and the $\mathrm{T} / \mathrm{D}$ ratio). Our findings suggest that task complexity affects spatial attention, and that spatial bias should not be associated with a single factor.
We found no difference in the distribution patterns of hit rates between the two groups, nor any evidence that supports the lateral difference in visual attention between children with or without dyslexia. On the other hand, most participants started their initial responses three times more often on the left side than on the right side. This result is consistent with the previous findings (Wang, Huang, \& Huang, 2006) for the performance of children without learning disabilities.

Sireteanu et al. (2005) found that adults and schoolchildren without dyslexia showed significant leftward deviations in line-bisection tasks, but this pattern has not been found in children with dyslexia. They concluded that the significant left visual "minineglect" was linked to right parietal deficits found in dyslexic participants. We found no evidence to support the lateral difference in visual attention between children with and without dyslexia. Our cancellation tasks may not be a sensitive tool for detecting minimal lateralization differences. However, given the small test populations of the Sireteanu et al. (2005) study, further research on this topic is warranted.

\section{Conclusion}

Test sensitivity and administration time are important and need to be evaluated to lessen the load on testers and testees for clinical assessments. We built a computerized cancellation system with four different cancellation forms to investigate visuospatial attention in children with and without dyslexia. We found that T/D ratios had a strong effect on completion time and PQ score, and that they should be considered for test difficulty when designing visuospatial attention assessments. Our findings also revealed that tasks with more stimuli were challenging for accuracy, took more time, and were able to differentiate the two groups better, but that fewer stimuli may be too easy and thus not sensitive to minor attention problems.

For the two groups, there were no group differences in Forms 1, 2, and 3 for the hit rate, or in Form 2 for the completion time and PQ score. The dyslexic group achieved the same performance as the control group on Form 2. We also proposed that hit rate may not be a sensitive indicator of minor dysfunction in visuospatial attention when the number of stimuli is smaller (120 vs. 240 in this study). When using hit rate for outcome measures in a cancellation test with a sufficient time frame, the tester has to be aware of the condition "number of stimuli."

\section{AUTHOR NOTE}

This research was supported in part by Grant NSC94-2614-S-51-001 from the National Science Council (NSC), Taiwan. The authors thank the NSC for their financial support, and thank everyone involved in this study. The authors also thank the reviewers for their insightful comments and suggestions. Address correspondence to T.-Y. Wang, Department of Occupational Therapy, National Cheng Kung University, 1 University Road, Tainan 701, Taiwan (e-mail: michwang@mail.ncku.edu.tw).

\section{REFERENCES}

Ben-Yehudah, G., Sackett, E., Malchi-Ginzberg, L., \& Ahissar, M (2001). Impaired temporal contrast sensitivity in dyslexics is specific to retain-and-compare paradigms. Brain, 124, 1381-1395.

Byrd, D. A., Touradj, P., Tang, M. X., \& Manly, J. J. (2004). Can- 
cellation test performance in African American, Hispanic, and White elderly. Journal of the International Neuropsychological Society, 10, 401-411.

Casco, C., \& Prunetti, E. (1996). Visual search of good and poor readers: Effects with targets having single and combined features. Perceptual \& Motor Skills, 82, 1155-1167.

Casco, C., Tressoldi, P. E., \& Dellantonio, A. (1998). Visual selective attention and reading efficiency are related in children. Cortex, 34, 531-546.

Chatterjee, A., Thompson, K. A., \& Ricci, R. (1999). Quantitative analysis of cancellation tasks in neglect. Cortex, 35, 253-262.

Dawson, D. R., \& TANNER-CoHEN, C. (1997). Visual scanning patterns in an adult Chinese population: Preliminary normative data. Occupational Therapy Journal of Research, 17, 264-279.

Di Filippo, G., Brizzolara, D., Chilosi, A., De Luca, M., Judica, A. \& PECINI, C. (2005). Rapid naming, not cancelation speed or articulation rate, predicts reading in an orthographically regular language (Italian). Child Neuropsychology, 11, 349-361.

Diller, L., Ben-Yishay, Y., Gerstman, L. J., Goodkin, W., \& WeinBERG, J. (1974). Studies in cognition and rehabilitation in hemiplegia. New York: New York University Medical Center.

Dumont, R., Stevens, C., Dawson, M. M., Guare, R., \& Weiler, M. (2001). Obtained score differences on the Mesulam continuous performance test: A comparison between ADHD subjects and controls. Retrieved June 1, 2007, from http://alpha.fdu.edu/psychology/ Mesulam_article.htm.

Duncan, J., \& Humphreys, G. W. (1989). Visual search and stimulus similarity. Psychological Review, 96, 433-458.

Facoetti, A., Lorusso, M. L., Paganoni, P., Umiltà, C., \& Mascetti, G. G. (2003). The role of visuospatial attention in developmental dyslexia: Evidence from a rehabilitation study. Cognitive Brain Research, 15, 154-164.

FriedmanN, N., \& Nachman-KatZ, I. (2004). Developmental neglect dyslexia in a Hebrew-reading child. Cortex, 40, 301-313.

Gauthier, L., Dehaut, F., \& Joanette, Y. (1989). The Bells test: A quantitative and qualitative test for visual neglect. International Journal of Clinical Neuropsychology, 11, 49-54

GeldMacher, D. S. (1996). Effects of stimulus number and target-todistractor ratio on the performance of random array letter cancellation tasks. Brain \& Cognition, 32, 405-415.

GELDMACHER, D. S. (1998). Stimulus characteristics determine processing approach on random array letter-cancellation tasks. Brain \& $\mathrm{Cog}$ nition, 36, 346-354.

Geldmacher, D. S., \& Alhaj, M. (1999). Spatial aspects of letter cancellation performance in Arabic readers. International Journal of Neuroscience, 97, 29-39.

Geldmacher, D. S., Doty, L., \& Heilman, K. M. (1994). Spatial performance bias in healthy elderly subjects on a letter cancellation task. Neuropsychiatry, Neuropsychology, \& Behavioral Neurology, 7, 275-280.

Halligan, P., \& Marshall, J. (1992). Left visuo-spatial neglect: A meaningless entity? Cortex, 28, 525-535.

Halligan, P., Wilson, B., \& Cockburn, J. (1990). A short screening test for visual neglect in stroke patients. International Disability Studies, 12, 95-99.

Hari, R., Renvall, H., \& Tanskanen, T. (2001). Left minineglect in dyslexic adults. Brain, 124, 1373-1380.

HAWELKa, S., \& WimMER, H. (2005). Impaired visual processing of multi-element arrays is associated with increased number of eye movements in dyslexic reading. Vision Research, 45, 855-863.
Heiervang, E., \& Hugdahl, K. (2003). Impaired visual attention in children with dyslexia. Journal of Learning Disabilities, 36, 68-73.

Hogeboom, M., \& van Leeuwen, C. (1997). Visual search strategy and perceptual organization covary with individual preference and structural complexity. Acta Psychologica, 95, 141-164.

Iles, J., Walsh, V., \& Richardson, A. (2000). Visual search performance in dyslexia. Dyslexia, 6, 163-177.

Kiliç, B. G., Sener, S., Koçar, A. I., \& KaraKas, S. (2007). Multicomponent attention deficits in attention deficit hyperactivity disorder. Psychiatry \& Clinical Neurosciences, 61, 142-148.

LeZaK, M. D. (1995). Neuropsychological assessment (3rd ed.). New York: Oxford University Press.

Mesulam, M. M. (2000). Principles of behavior and cognitive neurology. New York: Oxford University Press.

Quintana, L. (1995). Evaluation of perception and cognition. In C. Trombly (Ed.), Occupational therapy for physical dysfunction (pp. 201-224). Baltimore: Williams \& Wilkins.

Roeltgen, D. P., \& Roeltgen, M. G. (1990). Asymmetric lateralized attention in children. Developmental Neuropsychology, 6, 25-37.

Ruff, R. M., Evans, R. W., \& Light, R. H. (1986). Automatic detection vs controlled search: A paper-and-pencil approach. Perceptual \& Motor Skills, 62, 407-416.

Scharroo, J., Stalmeier, P. F. M., \& Boselie, F. (1994). Visual search and segregation as a function of display complexity. Journal of General Psychology, 121, 5-17.

Shalev, L., \& Tsal, Y. (2003). The wide attentional window: A major deficit of children with attention difficulties. Journal of Learning Disabilities, 36, 517-527.

Sireteanu, R., Goebel, C., Goertz, R., \& Wandert, T. (2006). Do children with developmental dyslexia show a selective visual attention deficit? Strabismus, 14, 85-93.

Sireteanu, R., Goertz, R., Bachert, I., \& Wandert, T. (2005). Children with developmental dyslexia show a left visual "minineglect." Vision Research, 45, 3075-3082.

Stein, J., \& Walsh, V. (1997). To see but not to read; the magnocellular theory of dyslexia. Trends in Neurosciences, 20, 147-152.

Taroyan, N. A., Nicolson, R. I., \& FAwCETt, A. J. (2007). Behavioural and neurophysiological correlates of dyslexia in the continuous performance task. Clinical Neurophysiology, 118, 845-855.

Treisman, A. M., \& Gelade, G. (1980). A feature-integration theory of attention. Cognitive Psychology, 12, 97-136.

Trick, L. M., \& EnNs, J. T. (1998). Lifespan changes in attention: The visual search task. Cognitive Development, 13, 369-386.

Vidyasagar, T. R., \& PAMmer, K. (1999). Impaired visual search in dyslexia relates to the role of the magnocellular pathway in attention. NeuroReport, 10, 1283-1287.

Wang, T.-Y., Huang, H.-C., \& Huang, H. S. (2006). Design and implementation of cancelation tasks for visual search strategies and visual attention in school children. Computers \& Education, 47, 1-16.

Wasserman, J. D., Mitchell, J. H., \& Melrose, S. (1995). Development of visual search strategies in normal children. Archives of Clinical Neuropsychology, 10, 400-401.

Weintraub, S., \& Mesulam, M. M. (1988). Visual hemispatial inattention: Stimulus parameters and exploratory strategies. Journal of Neurology, Neurosurgery \& Psychiatry, 51, 1481-1488.

Williams, M. C., Brannan, J. R., \& Lartigue, E. K. (1987). Visual search in good and poor readers. Clinical Vision Sciences, 1, 367-371.

(Manuscript received May 10, 2008; revision accepted for publication November 13, 2008.) 